This study was limited to highly selected patients, and the use of triple-dose gadolinium to increase sensitivity might have increased detection of subclinical lesions. Nonetheless, the authors say that their results support the concept that MRI conversion occurs in the majority of CIS patients shortly after the clinical onset of symptoms.

Caroline Barranco

Original article Pestalozza IF et al. (2005) Monthly brain magnetic resonance imaging scans in patients with clinically isolated syndrome. Mult Scler 11: 390-394

\section{Recognizing the signs of right-hemispheric stroke}

Left-hemispheric and right-hemispheric strokes have different characteristic symptoms; for instance, left-hemispheric stroke can cause aphasia, whereas right-hemispheric stroke is associated with neglect. The symptoms of right-hemispheric stroke can be difficult to recognize, raising concerns about the adequacy of clinical management in this patient group. A recent German study has examined the impact of such symptom differences on patient treatment, particularly with regard to admission to hospital.

Foerch and colleagues carried out statistical analysis of data from 20,097 stroke patients (mean age 70 years) who had received inpatient care for their condition-patients had sustained a transient ischemic attack, a cerebral infarction or an intracerebral hemorrhage. The data included information on stroke side and localization, stroke severity and cause, vascular risk factors, and time between the onset of symptoms and admission to hospital.

Overall, 11,328 patients were diagnosed with a left-sided stroke, and 8,769 with a rightsided stroke $(P<0.0001)$. Fifty-eight percent of patients who received thrombolytic treatment had suffered a left-hemispheric stroke, and a larger proportion of left-hemispheric than righthemispheric stroke patients received treatment within 3 hours. Overall, more patients were admitted to hospital with left-hemispheric than with right-hemispheric stroke, yet there is no evidence that left-hemispheric stroke occurs more frequently in the general population.

The findings of this study indicate that the poorer recognition of symptoms associated with right-hemispheric stroke, in particular during the first few hours after an event, leads to an inequality in the medical treatment and management of patients with left-hemispheric and right-hemispheric stroke.

Christine Kyme

Original article Foerch C et al. (2005) Difference in recognition of right and left hemispheric stroke. Lancet 366: 392-393

\section{Prenatal exposure to antiepileptic drugs - risk factor for autism spectrum disorder}

Rasalam and colleagues have found evidence to implicate prenatal exposure to anticonvulsant medication in various neurodevelopmental disorders, and as a risk factor for the development of autism spectrum disorder (ASD).

In this 20-year, population-based study, 626 children born to 389 mothers who had been exposed to anticonvulsant medication, were identified from Aberdeen Maternity Hospital (Scotland, UK) records. Of these, 150 mothers and 260 children participated in this study.

In structured interviews, 26 children were reported by their parents to have social or behavioral difficulties. Of these, 11 children were diagnosed with an autistic disorder, and one was diagnosed with Asperger syndrome. In the 260 children exposed to antiepileptic drugs, the prevalence of ASD was calculated to be $4.6 \%$. Sodium valproate was the drug most likely to be associated with an ASD; $8.9 \%$ of children exposed to this drug were diagnosed with an autistic disorder or Asperger syndrome. Several of the children with social or behavioral difficulties, but no autism, showed signs of a communication disorder such as a delay in the acquisition of speech and language skills.

The authors note that clinicians and families involved in the use of antiepileptic drugs during pregnancy should be aware of the association between these drugs and ASDs. This association should be considered when selecting the treatment options that are best for the patient and their family.

Marie Lofthouse

Original article Rasalam AD et al. (2005) Characteristics of fetal anticonvulsant syndrome associated autistic disorder. Dev Med Child Neurol 47: 551-555 